\title{
Motivación hacia el ejercicio en estudiantes universitarios, diferencias demográficas
}

\author{
Motivation towards exercise in college students, demographic differences
}

\section{Pablo Gutiérrez-García ${ }^{1 *}$, Alan Silvestre Rocha Rascón ${ }^{1}$, Roberto Andrés González Fimbres $^{1}$, María Grethel Ramírez Siqueiros ${ }^{1}$, Eduardo Gómez Infante ${ }^{1}$}

${ }^{1}$ Universidad Estatal de Sonora

"Autor de correspondencia: Pablo Gutiérrez-García,pablo.gutierrez@ues.mx

\section{Resumen}

El estudio analizó la motivación hacia la práctica del ejercicio de estudiantes de la Licenciatura en Entrenamiento Deportivo (LED). Soportado por la Teoría de la autodeterminación (TAD; Deci \& Ryan, 1985; 2002), el objetivo fue comparar las regulaciones motivacionales hacia el ejercicio en función del sexo, situación laboral y semestre cursado en estudiantes universitarios. Se recolectaron datos de 260 estudiantes de ambos sexos (172 hombres y 88 mujeres), con edades entre 19 a 32 años $(M=22.11, D T=2.19)$, todos completaron el Cuestionario de Regulación de la Conducta en el Ejercicio (BREQ-3; Zamarripa et al., 2018). Se evidenció asociación $(p<.01)$, entre las regulaciones más autodeterminadas (intrínseca, integrada e identificada) con la regulación introyectada. Las regulaciones menos autodeterminadas (externa y no motivación) se asocian positivamente entre sí, y negativamente con las más autodeterminadas. Se observaron efectos pequeños pero significativos de los indicadores demográficos en las regulaciones motivacionales $(p<.05)$, hombres presentan mayores valores en las regulaciones integrada, identificada, introyectada y no motivación con respecto al género; estudiantes que trabajan presentan mayores valores en las regulaciones integrada e identificada con respecto a situación laboral; y estudiantes del segundo ciclo presentan mayores valores en la regulación introyectada con relación a ciclo cursado. En conclusión, se confirma la confiabilidad de la versión mexicana BREQ-3, apoyando la predicción del continuo de la TAD. El efecto de los indicadores demográficos pudiera estar mediado por premisas socioculturales, por lo que se sugiere continuar con una mayor investigación para obtener resultados concluyentes.

Palabras clave: Procesos motivacionales, autonomía, actividad física

Abstract

During the study, the motivation towards the exercise of students of the Sports Training Bachelor program (Licenciatura en Entrenamiento Deportivo, LED) was analyzed. Supported by the Selfdetermination Theory (SDT; Deci \& Ryan, 1985; 2002). The objective was to compare the motivational regulations towards exercise based on sex, work situation and semester in college students. Data was collected from 260 students of both sexes (172 men and 88 women), aged from 19 to 32 years $(M=$ 22.11, $S D=2.19$ ), all completed the Exercise Behavior Regulation Questionnaire (BREQ3; Zamarripa et al., 2018). An association ( $p$ $<.01)$ between the most self-determined regulations (intrinsic, integrated and identified) with introjected regulation was evidenced. The less self-determined regulations (external and non-motivational) are positively associated with each other, and negatively with the more self-determined. Small but significant effects of demographic indicators on motivational regulations ( $p$ $<.05)$, men present higher values in integrated, identified, introjected and nonmotivated regulations regarding sex; working 
students present higher values in integrated and identified regulations regarding employment status; and second cycle students present higher values in introjected regulation by semester cycle. In conclusion, reliability of the Mexican version of the BREQ-3 is confirmed, supporting the predictions of the SDT continuum. Demographic indicators effect may be mediated by sociocultural premises, so further research is suggested for conclusive results.

Key words: Motivational processes, autonomy, physical activity

\section{Introducción}

La promoción de la práctica de Ejercicio Físico (EF) en la población universitaria se considera una línea prioritaria a trabajar, especialmente en aquellos profesionales que van a tener una relación directa en su quehacer profesional con el desarrollo de hábitos saludables y más activos en la población infantil y juvenil (Práxedes et al., 2016).

Algunos estudios han incursionado en los procesos motivacionales asociados a la práctica de EF (Alías, Aguilar \& Rodríguez, 2017; Concha-Viera et al., 2017; GonzálezCutre, Sicilia \& Águila, 2011; GonzálezCutre, Sicilia, \& Fernández, 2010; McDavid, Cox, \& McDonough, 2014; Mazorra, Oliva, \& Palmeira, 2020; Práxedes et al., 2016; Román et al., 2018; Wilson et al., 2006; Zamarripa et al., 2018). La motivación se entiende como la causa psicológica de cualquier acción, demostrándose como una variable que correlaciona positivamente y regula las conductas hacia el EF (Martínez, Mayorga, \& Viciana, 2018). Se ha demostrado que la motivación que tienen las personas puede ser un factor predominante para lograr cualquier meta, por lo cual la falta de motivación puede estar implicada en la falta de ejercicio, principalmente los jóvenes por la falta de motivación observada en la etapa posterior a la adolescencia que puede generar el abandono o la ausencia de adherencia a la práctica de EF afectando su calidad de vida y/o estado de salud (MolinaGarcía, Castillo, \& Pablos, 2009; Wang et al., 2009).

Una de las teorías motivacionales que nos ayuda a comprender el funcionamiento de la motivación en los seres humanos en contextos sociales es la Teoría de la Autodeterminación (Self-determination Theory, SDT; Deci \& Ryan, 1985; 2002). La Teoría de la Autodeterminación (TAD) se centra principalmente en el nivel psicológico y diferencia los tipos de motivación a lo largo de un continuo de autodeterminación (Deci \& Ryan, 1985; 2000; Ryan \& Deci, 2000). Deci \& Ryan (1985) plantean que en los extremos opuestos del continúo planteado por la TAD se encuentran la motivación intrínseca y la no motivación, y entre ellas se encuentran otros tipos de motivación con mayor o menor grado de la misma (regulación externa, regulación introyectada, regulación identificada y regulación integrada).

La motivación intrínseca se considera la forma de motivación más autodeterminada, presentándose cuando una persona realiza $\mathrm{EF}$ por el placer de experimentarlo, además, del disfrute y la satisfacción, sin esperar una recompensa o tratando de evitar el castigo. Por otro lado, al final del continuo se encuentra la no motivación (forma menos autodeterminada), reflejándose la falta de intención de comprometerse, donde la persona no tiene ninguna motivación para participar en la realización de EF (Deci \& Ryan, 2000). Las personas que no están motivadas para realizar EF y deporte no buscan objetivos sociales, afectivos $\mathrm{O}$ materiales; en cambio, ellos experimentan sensaciones negativas como incompetencia, apatía, e incluso depresión (Vallerand, 2001). 
La TAD, está compuesta entre otras por una mini-teoría llamada teoría de integración organísmica (Deci \& Ryan, 1985), la misma se introdujo para explicar las diferentes formas de motivación extrínseca y los factores contextuales que promueve la internalización y la integración de comportamientos de regulación. Esta teoría propone un orden o secuencia para el grado de autonomía con el que se regula la motivación extrínseca. La secuencia consta de regulación integrada, identificada, introyectada y regulación externa. La regulación externa es la menos autónoma del tipo de motivación extrínseca, e incluye el caso clásico de motivación para obtener recompensas o evitar el castigo. La regulación introyectada está algo más internalizada y basada en comportamientos llevados a cabo para evitar la culpa, la ansiedad y la vergüenza, o para mejorar el ego, los sentimientos de valor o el orgullo. La regulación identificada implica otorgar un valor consciente a un comportamiento de tal manera que la acción se acepta cuando es personalmente importante (Ryan \& Deci, 2000). Por último, la regulación integrada representa la forma más autónoma de motivación extrínseca, que ocurre cuando se han identificado las regulaciones, evaluado y asimilado (Ryan \& Deci, 2000).

La población universitaria es considerada la población adulto joven clave para la promoción y prevención de salud para las futuras generaciones, por lo que es trascendental identificar entre otras situaciones actuales, la motivación hacia el EF de los universitarios (Alías et al., 2017; Mazorra, Oliva, \& Palmeira, 2020; Rodríguez-Martín et al., 2019). En este sentido Plotnikoff et al. (2015) plantean que esta etapa universitaria es un periodo clave para la promoción del EF, lo cual se mostró en población española que está cursando estudios universitarios, vinculados con la formación en hábitos saludables.

En su estudio Práxedes et al. (2016) encontraron que los estudiantes de titulaciones directamente relacionadas con la práctica físico deportiva presentaron valores superiores, en las regulaciones motivacionales hacia la actividad física más autodeterminadas (motivación intrínseca, regulación integrada y regulación identificada). Debido a ello, concluyeron que la motivación puede ser una variable influyente en la intención de ser físicamente activo en la etapa universitaria. En este sentido, si el programa de formación profesional tiene un vínculo estrecho con el $\mathrm{EF}$, los estudiantes encontrarán más divertida la práctica físico deportiva, valorando los beneficios y sentirán una mayor conexión con su propio estilo de vida.

Las investigaciones han incursionado principalmente en las diferencias de las regulaciones motivacionales hacia la práctica de EF en función del género (GonzálezCutre, Sicilia \& Águila, 2011; Navarro et al., 2008; Román et al., 2018). Los resultados de González-Cutre et al. (2011) y Navarro et al. (2008) fueron similares encontrando que las mujeres presentaron valores más elevados que los hombres en las formas de motivación más autodeterminadas. Sin embargo, en su estudio Román et al. (2018) rechazó la hipótesis planteada de que los hombres mostrarían valores más elevados que las mujeres en las formas de motivación más autodeterminadas; encontrando medias más altas solamente en las regulaciones introyectada y regulación extrínseca.

Reconociendo la importancia que tiene la motivación en la realización de EF entre los universitarios cuya formación está relacionada con la práctica físico deportiva, se considera necesario identificar el tipo de motivación que prevalece en esta población, así como identificar si ésta es diferenciada en 
función de datos demográficos. Por otra parte, es importante la aplicación del cuestionario (Regulación de la Conducta en el Ejercicio, BREQ-3; Zamarripa et al., 2018) validado en población mexicana, aumentando con ello la generación de conocimiento y producción científica en el contexto universitario.

Por ello el objetivo del estudio fue examinar la consistencia interna del BREQ-3 y comparar las regulaciones motivacionales de estudiantes universitarios del área del entrenamiento deportivo en función del sexo, situación laboral actual y ciclo cursado, en este sentido el primer ciclo está integrado por alumnos de los semestres segundo y cuarto, mientras que el segundo ciclo está integrado por alumnos de los semestres sexto y octavo.

\section{Método}

\section{Diseño}

El estudio cuenta con un diseño transversal con alcance descriptivo y correlacional (Hernández, Fernández, \& Baptista, 2014).

\section{Participantes}

La población estuvo conformada por universitarios que cursaban la Licenciatura en Entrenamiento Deportivo (LED) en la Universidad Estatal de Sonora (UES) en México. Mediante un muestreo por conveniencia, participaron en la investigación alumnos oficialmente inscritos en el semestre en curso, mientras que los estudiantes que no estuvieran inscritos oficialmente no podían participar.

La muestra de estudio la conformaron 260 jóvenes de ambos sexos (172 hombres y 88 mujeres), con edades entre 19 a 32 años $(M=22.11, D T=2.19)$; con base a la situación laboral en el semestre 130 estudiantes si trabajan y 130 no trabajan; y respecto al ciclo cursado se consideró (Primer ciclo son 102 estudiantes de segundo y cuarto semestres y Segundo ciclo son 158 estudiantes de sexto y octavo semestres).

\section{Instrumento}

Para evaluar las regulaciones motivacionales se utilizó el Cuestionario de Regulación de la Conducta en el Ejercicio (BREQ-3), el cual mide la forma de motivación más autodeterminada a la menos autodeterminada, validado con población mexicana (Zamarripa et al., 2018). El BREQ3 es un cuestionario de 23 ítems dividido en seis subescalas: regulación intrínseca (cuatro elementos; por ejemplo, "... porque creo que el ejercicio es divertido"), regulación integrada (cuatro elementos; por ejemplo, "... porque concuerda con mi forma de vida"), regulación identificada (tres ítems; por ejemplo, "... porque valoro los beneficios que tiene el ejercicio físico"), regulación introyectada (cuatro elementos; por ejemplo, "... porque me siento culpable cuando no lo hago"), regulación externa (cuatro elementos; por ejemplo, "... porque los demás me dicen que debo hacerlo"), y no motivación (cuatro elementos; por ejemplo, ... pero no veo el sentido de hacer ejercicio"). El cuestionario comienza con la raíz "Yo hago ejercicio físico ... ", las respuestas se dan en una escala de tipo Likert de 5 puntos que van desde 0 (definitivamente no es cierto) a 4 (definitivamente cierto), los valores de respuesta de cada variable psicológica se promedian. Investigaciones anteriores han confirmado la confiabilidad de este instrumento (González-Cutre et al., 2010; Wilson et al., 2006).

\section{Procedimiento}

Para llevar a cabo el estudio se solicitó la aprobación a la UES a través de la jefatura de carrera de LED, así como el consentimiento de participación de los estudiantes. Se utilizó la plataforma de Google Forms para realizar la captura del 
cuestionario BREQ-3 en línea para que fuera requisitado por los alumnos y posteriormente los datos recolectados se vaciaron en una hoja de cálculo de Excel para su análisis.

\section{Análisis de datos}

Los datos fueron importados para su análisis al paquete estadístico SPSS versión 23. Se utilizó la estadística descriptiva de cada variable y por ítem mediante las medidas de tendencia central (moda, mediana, media, asimetría y curtosis), así como el cálculo de normalidad de los datos a través de la prueba de Kolmogorov-Smirnov; la fiabilidad mediante el coeficiente alpha de Cronbach, donde el punto de corte utilizado fue de .70 propuesto por Nunnally (1978). Las correlaciones entre los factores de las regulaciones motivacionales se analizaron a través del Coeficiente de Spearman. Para el análisis estadístico inferencial se utilizó la prueba no paramétrica $U$ de Mann-Whitney de comparación de dos muestras independientes. Además, para calcular el tamaño del efecto se utilizó la prueba estadística Hedges.

\section{Resultados}

En la Tabla 1 se presentan los estadísticos descriptivos de cada uno de los 23 ítems del cuestionario BREQ-3, mostrando la media $(M)$, desviación típica (DT), asimetría y curtosis, en el cual los datos indican que el ítem 18 en la pregunta "Yo hago ejercicio físico porque encuentro el ejercicio una actividad agradable" tiene la media más alta $(M=3.64)$ contando con una asimetría de -2.05 (siendo la asimetría más baja de los ítems) y una curtosis de 4.51; mientras que le media más baja es para el ítem 23 "Yo hago ejercicio físico pero pienso que hacer ejercicio es una pérdida de tiempo" $(M=.26)$ contando con una asimetría de 3.42 y una curtosis de 11.72 positiva fuerte (siendo la curtosis más alta de los ítems).

Así mismo los estadísticos indican que no hay normalidad en los ítems ya que se encuentran fuera del rango de los valores de asimetría y curtosis.

En la Tabla 2 se muestran los ítems que corresponden a cada subescala del BREQ-3, media $(M)$, desviación típica $(D T)$, Kolmogorov-Smirnov $(K-S)$ para pruebas no paramétricas que arrojó como resultado que la distribución no es normal, de igual manera podemos observar que la regulación intrínseca presenta la media más alta $(M=$ 14.03) siguiendo con la regulación integrada e identificada con una media de $(M=12.66)$ y $(M=10.40)$ respectivamente, por otro lado, la media más baja fue para la regulación externa $(M=1.75)$. El resultado de la fiabilidad fue analizado mediante el coeficiente Alpha de Cronbach $(\alpha)$ con valores entre (.84 a .70).

En la Tabla 3 se muestra las correlaciones de las subescalas del BREQ-3, para obtener los resultados se utilizó la prueba estadística de correlaciones de Spearman. El grado de relación positiva de las subescalas $(p<.01)$ entre la regulación integrada respecto a la regulación intrínseca $(r=.67)$, de igual manera positivamente con la regulación identificada $(r=.68)$. La regulación intrínseca se asocia de forma negativa con la regulación externa $(r=-23)$ y la no motivación ( $r=-.27$ ) y éstas a su vez se asocian negativamente con la regulación identificada $(r=-.23)$ y $(r=-.24)$ respectivamente. 


\section{Tabla 1}

Estadísticos descriptivos de los ítems del BREQ-3

\begin{tabular}{|c|c|c|c|c|c|}
\hline Ítems & & $M$ & $D T$ & Asimetría & Curtosis \\
\hline 1. & $\begin{array}{l}\text { Yo hago ejercicio físico porque los demás me dicen } \\
\text { que debo hacerlo. }\end{array}$ & .45 & .938 & 2.30 & 4.77 \\
\hline 2. & $\begin{array}{l}\text { Yo hago ejercicio físico porque me siento culpable } \\
\text { cuando no lo hago. }\end{array}$ & 1.39 & 1.48 & .58 & -1.10 \\
\hline 3. & $\begin{array}{l}\text { Yo hago ejercicio físico porque valoro los beneficios } \\
\text { que tiene el ejercicio físico }\end{array}$ & 3.61 & .73 & -2.06 & 4.51 \\
\hline 4. & $\begin{array}{l}\text { Yo hago ejercicio físico porque creo que el ejercicio es } \\
\text { divertido }\end{array}$ & 3.52 & .87 & -2.00 & 3.87 \\
\hline & $\begin{array}{l}\text { Yo hago ejercicio físico porque concuerda con mi } \\
\text { forma de vida }\end{array}$ & 3.41 & .95 & -1.72 & 2.56 \\
\hline & $\begin{array}{l}\text { Yo hago ejercicio físico, pero no sé por qué tengo que } \\
\text { hacerlo }\end{array}$ & .64 & 1.16 & 1.84 & 2.26 \\
\hline & $\begin{array}{l}\text { Yo hago ejercicio físico porque mis } \\
\text { amigos/familia/pareja me dicen que debo hacerlo }\end{array}$ & .50 & 1.04 & 2.11 & 3.45 \\
\hline & $\begin{array}{l}\text { Yo hago ejercicio físico porque me siento avergonzado } \\
\text { si falto a la sesión }\end{array}$ & .73 & 1.21 & 1.50 & .97 \\
\hline & $\begin{array}{l}\text { Yo hago ejercicio físico porque para mí es importante } \\
\text { hacer ejercicio regularmente }\end{array}$ & 3.44 & .97 & -1.92 & 3.19 \\
\hline & $\begin{array}{l}\text { Yo hago ejercicio físico porque considero que el } \\
\text { ejercicio físico forma parte de mi ser }\end{array}$ & 3.20 & 1.10 & -1.35 & .99 \\
\hline & $\begin{array}{l}\text { Yo hago ejercicio físico, pero no sé por qué me molesto } \\
\text { en hacer ejercicio físico }\end{array}$ & .72 & 1.16 & 1.49 & 1.05 \\
\hline & $\begin{array}{l}\text { Yo hago ejercicio físico porque disfruto con las } \\
\text { sesiones de ejercicio }\end{array}$ & 3.46 & .93 & -1.95 & 3.63 \\
\hline & $\begin{array}{l}\text { Yo hago ejercicio físico porque otras personas se } \\
\text { enojarían conmigo si no hago ejercicio }\end{array}$ & .32 & .88 & 3.07 & 8.91 \\
\hline & $\begin{array}{l}\text { Yo hago ejercicio físico, pero no veo el sentido de } \\
\text { hacer ejercicio }\end{array}$ & .41 & .98 & 2.54 & 5.64 \\
\hline & $\begin{array}{l}\text { Yo hago ejercicio físico porque veo el ejercicio físico } \\
\text { como una parte fundamental de lo que soy }\end{array}$ & 3.16 & 1.12 & -1.17 & .44 \\
\hline & $\begin{array}{l}\text { Yo hago ejercicio físico porque siento que he fallado } \\
\text { cuando dejo de hacer ejercicio por un tiempo }\end{array}$ & 1.95 & 1.58 & .01 & -1.53 \\
\hline & $\begin{array}{l}\text { Yo hago ejercicio físico porque pienso que es } \\
\text { importante hacer el esfuerzo de ejercitarme } \\
\text { regularmente }\end{array}$ & 3.35 & .95 & -1.48 & 1.62 \\
\hline 18. & $\begin{array}{l}\text { Yo hago ejercicio físico porque encuentro el ejercicio } \\
\text { una actividad agradable }\end{array}$ & 3.64 & .74 & -2.44 & 6.66 \\
\hline & $\begin{array}{l}\text { Yo hago ejercicio físico porque me siento presionado } \\
\text { por mis amigos/familia para realizar ejercicio }\end{array}$ & .48 & 1.08 & 2.25 & 3.89 \\
\hline & $\begin{array}{l}\text { Yo hago ejercicio físico porque considero que el } \\
\text { ejercicio físico concuerda con mis valores }\end{array}$ & 2.89 & 1.30 & -.99 & -.15 \\
\hline & $\begin{array}{l}\text { Yo hago ejercicio físico porque me pongo nervioso si } \\
\text { no hago ejercicio regularmente }\end{array}$ & .87 & 1.33 & 1.32 & .40 \\
\hline & $\begin{array}{l}\text { Yo hago ejercicio físico porque hacer ejercicio me } \\
\text { resulta placentero y satisfactorio }\end{array}$ & 3.41 & .97 & -1.79 & 2.63 \\
\hline 23. & $\begin{array}{l}\text { Yo hago ejercicio físico, pero pienso que hacer } \\
\text { ejercicio es una pérdida de tiempo }\end{array}$ & .26 & .79 & 3.42 & 11.72 \\
\hline
\end{tabular}

Nota: $M=$ Media; $D T=$ Desviación Típica 
Tabla 2

Estadísticos descriptivos, normalidad y consistencia interna de las subescalas del BREQ-3

\begin{tabular}{llllll}
\hline Variable & \multicolumn{1}{c}{ tems } & $\boldsymbol{M}$ & $\boldsymbol{D T}$ & $\boldsymbol{K}-\boldsymbol{S}$ & $\boldsymbol{\alpha}$ \\
\hline Regulación intrínseca & $4,12,18,22$ & 14.03 & 2.76 & $.26^{*}$ & .79 \\
Regulación integrada & $5,10,15,20$ & 12.66 & 3.70 & $.18^{*}$ & .84 \\
Regulación identificada & $3,9,17$ & 10.40 & 2.09 & $.23^{*}$ & .68 \\
Regulación introyectada & $2,8,16,21$ & 4.95 & 4.07 & $.11^{*}$ & .70 \\
Regulación externa & $1,7,13,19$ & 1.75 & 2.97 & $.32^{*}$ & .74 \\
No motivación & $6,11,14,23$ & 2.03 & 3.26 & $.29^{*}$ & .79
\end{tabular}

Nota: $K-S=$ Kolmogorov y Smirnov, $\alpha=$ Alfa de Cronbach.

En cuanto al grado de relación entre la regulación integrada y la regulación identificada se asocia positivamente $(r=.67)$, de igual manera la regulación integrada se asocia de manera positiva con la regulación regulación identificada se asocia de forma positiva con la regulación introyectada $(r=$ $.15, p<.05)$. La regulación externa se asocia de manera positiva con la no motivación $(r=$ $.37, p<.01)$. introyectada $(r=.21)$. Por otra parte, la

Tabla 3

Resultados de correlaciones de las subescalas del BREQ-3

\begin{tabular}{llllll} 
Variable & $\mathbf{1}$ & $\mathbf{2}$ & $\mathbf{3}$ & $\mathbf{4}$ & $\mathbf{5}$ \\
2. Regulación integrada & $.67^{* *}$ & -- & & & \\
3. Regulación identificada & $.68^{* *}$ & $.67^{* *}$ & -- & & \\
4. Regulación introyectada & .05 & $.21^{* *}$ & $.15^{*}$ & -- & \\
5. Regulación externa & $-.23^{* *}$ & -.07 & $-.23^{* *}$ & $.42^{* *}$ & - \\
6. No motivación & $-.27^{* *}$ & -.10 & $-.24^{* *}$ & $.37^{* *}$ & $.59^{* *}$ \\
\hline
\end{tabular}

Nota: 1 Regulación intrínseca. ${ }^{* *} p<.01,{ }^{*} p<.05$.

En la Tabla 4 se observan los resultados de las pruebas estadísticas no paramétricas para analizar las diferencias entre las variables psicológicas y variables demográficas las cuales mostraron que, con relación al sexo, los hombres presentan valores mayores de regulación integrada ( $g=$ $0.44, p<.05)$, identificada $(g=0.23, p<.05)$ e introyectada $(g=0.28, p<.05)$, así como de no motivación $(g=0.27, p<.05)$, sin embargo, con tamaños de efecto pequeños. Aunque se observó significancia en las diferencias entre estudiantes que trabajan y que no trabajan de regulación integrada e identificada, el análisis muestra que los tamaños de efecto son triviales $(g=0.01 \mathrm{y} g$ $=0.00$ respectivamente). Con relación al ciclo cursado, se observó diferencia pequeña solamente en regulación introyectada ( $g=$ $0.26, p<.05)$.

\section{Discusión}

El objetivo de este estudio fue comparar las regulaciones motivacionales hacia el ejercicio en función del sexo, situación laboral y ciclo cursado en estudiantes universitarios de LED-UES. Como resultado importante se muestra que el análisis de consistencia interna de las sub escalas del Cuestionario de Regulación de la conducta en el ejercicio, BREQ-3 presentó resultados adecuados en las subescalas, lo cual va en línea con lo planteado por varios autores del instrumento BREQ-3 (GonzálezCutre et al., 2010; Práxedes et al., 2016; Zamarripa et al., 2018). Lo anterior confirma la aceptación y utilización del instrumento de evaluación (BREQ-3). 
Los resultados del estudio muestran asociación entre las regulaciones más autónomas (intrínseca, integrada e identificada). De igual manera las tres regulaciones más autodeterminadas se asocian positivamente, aunque con valores leves con la regulación introyectada lo cual está alineado a los resultados encontrados en el estudio (Zamarripa et al., 2018). En este sentido, los resultados del presente estudio muestran relaciones de manera significativa específicamente con las regulaciones integrada e identificada. Por el contrario, las regulaciones externa y no motivación se relacionan positiva y significativamente entre ellas, y de manera negativa con las regulaciones más autónomas anteriormente mencionadas. Estos resultados están alineados con los encontrados por Zamarripa et al. (2018), mismos que refuerzan la predicción hecha por Deci \& Ryan (1985) sobre el continuo planteado por la teoría de la autodeterminación. Los factores que se encuentran en los extremos del continuo correlacionan de manera positiva y con resultados más elevados, mismos que también obtuvieron con muestras e idiomas diferentes en investigaciones (GonzálezCutre, Sicilia, \& Fernández, 2010; Wilson et al., 2006). Desde un punto de vista práctico, se hace énfasis en la necesidad de fomentar ambientes motivadores más autónomos en los jóvenes, dando como beneficios experiencias positivas, mayor compromiso y disfrute, así como una mayor adherencia a la práctica de EF (Alías, Aguilar \& Rodríguez, 2017).

Tabla 4

Análisis inferencial de las regulaciones motivacionales por sexo, situación laboral y ciclo cursado

\begin{tabular}{lllllll}
\hline & SEXO & \multicolumn{3}{c}{$\begin{array}{l}\text { SITUACIÓN } \\
\text { LABORAL }\end{array}$} & \multicolumn{2}{c}{$\begin{array}{l}\text { CICLO } \\
\text { CURSADO }\end{array}$} \\
\hline $\begin{array}{l}\text { Regulaciones } \\
\text { motivacionales }\end{array}$ & Hombre & Mujer & $\mathrm{Si}$ & No & $\begin{array}{l}\text { Primer } \\
\text { ciclo }\end{array}$ & $\begin{array}{l}\text { Segundo } \\
\text { ciclo }\end{array}$ \\
& & & & & & \\
& & & & & & \\
& $(\mathrm{n}=172)$ & $(\mathrm{n}=88)$ & $\mathrm{n}=130)$ & $(\mathrm{n}=130)$ & $(\mathrm{n}=102)$ & $(\mathrm{n}=158)$ \\
Intrínseca & $M \pm D T$ & $M \pm D T$ & $M \pm D T$ & $M \pm D T$ & $M \pm D T$ & $M \pm D T$ \\
Integrada & $3.56 \pm .62$ & $3.40 \pm .80$ & $3.50 \pm .72$ & $3.51 \pm .66$ & $3.48 \pm .73$ & $3.53 \pm .66$ \\
Identificada & $3.30 \pm .85^{*}$ & $2.90 \pm 1.01$ & $3.28 \pm .91^{*}$ & $3.05 \pm .93$ & $3.16 \pm .86$ & $3.17 \pm .97$ \\
Introyectada & $3.52 \pm .67^{*}$ & $3.36 \pm .74$ & $3.53 \pm .69^{*}$ & $3.39 \pm .70$ & $3.41 \pm .77$ & $3.50 \pm .65$ \\
Externa & $1.33 \pm 1.03^{*}$ & $1.05 \pm .97$ & $1.30 \pm 1.08$ & $1.17 \pm .95$ & $1.08 \pm 1.02$ & $1.34 \pm .1 .00^{*}$ \\
No motivación & $.46 \pm .72$ & $.39 \pm .78$ & $.41 \pm .71$ & $.46 \pm .77$ & $.32 \pm .66$ & $.51 \pm .78$ \\
& $.58 \pm .85^{*}$ & $.36 \pm .73$ & $.50 \pm .79$ & $.52 \pm .83$ & $.42 \pm .76$ & $.56 \pm .85$
\end{tabular}

$* p<.05$.

En relación a las diferencias en cuanto al sexo se evidencia en dos de las regulaciones más autónomas (integrada e identificada), las cuales presentan valores más elevados en los hombres que en las mujeres. Estos resultados están en consonancia con lo planteado por Concha-
Viera y Colaboradores (2017) donde únicamente en la regulación integrada se muestran diferencias significativas con valores más altos en los hombres. La explicación de estas diferencias pudiera estar dada por premisas socioculturales en población mexicana, debido a que culturalmente los hombres se caracterizan 
por prácticas de EF más dinámicas y activas que las mujeres. Por otra parte, los resultados son similares a los obtenidos en el estudio de Román et al. (2018) donde la regulación introyectada puntea más alto en los hombres que en las mujeres.

En cuanto a la situación laboral que presentan los estudiantes en cuanto a si están o no trabajando, se muestran diferencias en cuanto a las regulaciones integrada $\mathrm{e}$ identificada con valores más altos en los estudiantes que trabajan. Los resultados mostrados se alinean con Mazorra, Oliva, \& Palmeira (2020) los cuales encontraron diferencias interesantes en los estudiantes que realizan actividad laboral. Este indicador sociodemográfico determinante para la práctica de $\mathrm{EF}$, es considerada como una actividad extracurricular, que implica planear y distribuir adecuadamente el tiempo. En la misma línea de la actividad laboral Sousa, Fonseca y Barbosa (2013) con estudiantes universitarios brasileños, muestran como el exceso de trabajo, representado en horas dedicadas a la semana, es una de las barreras situacionales más prevalentes para esta población y que afecta la destinación de tiempo para el desarrollo del EF.

Finalmente, se encontraron diferencias por ciclo cursado mostrando valores más elevados los estudiantes de los últimos dos años que cursan la carrera de LED en la regulación introyectada. En este sentido, no se encontraron estudios con resultados similares, sin embargo, consideramos que los mismos pudieran estar dados por la necesidad de sobresalir profesionalmente para poder ser contratado al egresar de sus estudios universitarios. El desarrollo de los procesos motivacionales de tipo más autónomo son una prioridad para el profesional que tendrá una relación directa en su quehacer, siendo fundamental para la transmisión de patrones de conducta activos y duraderos en la sociedad.

\section{Conclusiones}

En conclusión, los resultados obtenidos en el estudio confirman que la versión mexicana del BREQ-3 es confiable para el estudio de las regulaciones motivacionales en el ejercicio. Por otra parte, se confirma la predicción de la TAD acerca del continuo de autodeterminación. Los resultados mostraron que los factores cercanos correlacionan de manera positiva significativa entre ellos y de manera contraria con los que están en el extremo del continuo.

Los estudiantes universitarios tanto hombres como mujeres presentan motivaciones diferentes ante el EF durante su proceso de formación profesional. Además, las formas de motivación más autodeterminadas se irán desarrollando por la necesidad e importancia de la práctica del EF independientemente de su situación laboral. Finalmente, se muestra que las conductas de los estudiantes, evidenciadas en los últimos semestres son guiadas por sentimientos de obligación interno hacia el EF.

Algunas limitaciones en el estudio están dadas por el tamaño de la muestra la cual se considera debe incrementarse en futuras investigaciones, así como la homogeneidad en la cantidad de estudiantes según el sexo. Se sugieren, estudios longitudinales para entender las diferencias que puedan existir en varios momentos en las variables de estudio.

Desde un punto de vista práctico, el estudio contribuye al área de formación de profesionales de la salud con aporte de conocimiento a los estudiantes, maestros y directivos, mostrando la necesidad del desarrollo de hábitos saludables y activos en este grupo de población.

\section{Referencias}

Alías, A., Aguilar, J. M., \& Rodríguez, A. I. H. (2017). Motivaciones de los estudiantes universitarios ante la 
práctica de actividad físico-deportiva de tiempo libre. Las actividades náuticas. Psychology, Society, \& Education, 8(3), 229-242.

Concha-Viera, A. M., Cuevas-Ferrer, R., Campos-Romero, P., \& GonzálezHernández, J. (2017). Recursos motivacionales para la autorregulación en la actividad física en edad universitaria. Cuadernos de Psicología del Deporte, 17(2), 27-34.

Deci, E. L., \& Ryan, R. M. (1985). Intrinsic motivation and selfdetermination in human behavior. New York, N.Y.: Plenum Press.

Deci, E. L., \& Ryan, R. M. (2000). The "what" and "why" of goal pursuits: Human needs and the selfdetermination of behavior. Psychological Inquiry, 11(4), 227268.

Deci, E., \& Ryan, R. (2002). Handbook of Self-Determination Research. New York, NY: The University Rochester Press.

González-Cutre, D., Sicilia, A., \& Águila, C. (2011). Interplay of different contextual motivations and their implications for exercise motivation. Journal of Sport Science and Medicine, 10(2), 274-282.

González-Cutre, D., Sicilia, A., \& Fernández, A. (2010). Hacia una mayor comprensión de la motivación en el ejercicio físico: medición de la regulación integrada en el contexto. Psicothema 22, 841-847.

Hernández, R., Fernández, C., \& Baptista, P. (2014). Metodología de la investigación. (6ta ed.). México, D. F.: McGraw-Hill.

Martínez, A., Mayorga, D. \& Viciana, J. (2018). Factores predictores de la actividad física en escolares españoles de acuerdo a su estado de peso. Retos, 33, 74-80.

Mazorra, M. G., Oliva, D. S., \& Palmeira, A. (2020). Actividad física en tiempo libre en estudiantes universitarios colombianos. Retos: nuevas tendencias en educación física, deporte y recreación, 37, 181189.

McDavid, L., Cox, A.E., \& McDonough, M.H. (2014). Need fulfillment and motivation in physical education predict trajectories of change in leisure-time physical activity in early adolescence. Psychology of Sport and Exercise, 15(5), 471-480. doi:10.1016/j.psychsport.2014.04.00 6

Molina-García, J., Castillo, I., \& Pablos, C. (2009). Determinants of leisuretime physical activity and future intention to practice in Spanish college students. The Spanish Journal of Psychology, 12(1), 128-137.

Navarro, N., González-Cutre, D., Marcos, P. J., Borges, F., Hernández, A., Vera, J. A., \& Moreno, J. A. (2008). Perfiles motivacionales en la actividad física saludable: un estudio desde la perspectiva de la teoría de la autodeterminación. En Actas del XI Congreso Nacional, XI Andaluz y III Iberoamericano de Psicología de la Actividad Física y del Deporte. Sevilla: Universidad Pablo de Olavide.

Nunnally, J. C. (1978). Psychometric theory (2nd ed.) New York: McGrawHill.

Plotnikoff, R.E., Costigan, S.A., Williams, R.L., Hutchesson, M.J., Kennedy, S.G., Robards, S.L., Allen, J., Collins, C. E., Callister, R., \& Germov, J. (2015). Effectiveness of interventions targeting physical activity, nutrition and healthy weight 
for university and college students: a systematic review and meta-analysis. International Journal of Behavioral Nutrition and Physical Activity, 12, 45. doi: 10.1186/s12966-015-0203-7

Práxedes, A., Sevil, J., Moreno, A., Del Villar, F., \& García-González, L. (2016). Niveles de actividad física y motivación en estudiantes universitarios. Diferencias en función del perfil académico vinculado a la práctica físicodeportiva. Journal of Sport and Health Research, 8(3), 191-204.

Rodríguez-Martín, A., Cano, M. S., Jiménez-Rodríguez, A., \& NovalbosRuiz, J. P. (2019). Educando con el ejemplo. Alimentación y actividad física en los futuros promotores de salud. Rev Esp Nutr Comunitaria, 25(4), 172-178.

Román, M. L., Matador, J. G., García, J. P. F., \& Castuera, R. J. (2018). Análisis de variables motivacionales y de estilos de vida saludables en practicantes de ejercicio físico en centros deportivos en función del género. Retos: nuevas tendencias en educación física, deporte $y$ recreación, 34, 166-171.

Ryan, R. M., \& Deci, E. L. (2000). Teoría de la autodeterminación y facilitación de la motivación intrínseca, el desarrollo social y el bienestar. Psicólogo estadounidense, $55,68-78$.
Sousa, T. F., Fonseca, S. A., \& Barbosa, A. R. (2013). Perceived barriers by university students in relation the leisuretime physical activity. Brazilian Journal of Kinanthropometry and Human Performance, 15(2), 164- 173. https://doi.org/10.5007/19800037.20 13v15n2p164

Wang, D., Ou, C.Q., Chen, M.Y., \& Duan, N. (2009). Health-promoting lifestyles of university students in Mainland China. BMC Public Health, 9(379). doi:10.1186/1471-2458-9379

Vallerand, R. J. (2001). "A hierarchical model of intrinsic and extrinsic motivation in sport and exercise settings," in Advances in Motivation in Sport and Exercise (Champaign, IL: Human Kinetics), 321-357.

Wilson, P.M., Rodgers, W.M., Loitz, C.C., \& Scime, G. (2006). «It's who I am ... really!». The importance of integrated regulation in exercise contexts. Journal of Applied Biobehavioral Research, 11, 79-104. Zamarripa, J., Castillo, I., Baños, R., Delgado, M., \& Álvarez, O. (2018). Motivational Regulations Across the Stages of Change for Exercise in the General Population of Monterrey (Mexico). Frontiers. Psychology, 9:2368.

DOI: 10.3389/fpsyg.2018.02368 